${ }^{4}$ Hardy JD, Solomon S, Banwell GS, Beach R, Wright V, Howard FM. Congenital complete heart block in the newborn associated with maternal systemic lupus erythematosus and other connective tissue antibodies. Arch Dis Child 1979;54: $7-13$.

5 Lanham JG, Walport MJ, Hughes GRV. Congenital heart block and familial connective tissue disease. $J$ Rheumatol 1983;10: 823-5.

\footnotetext{
6 Jacksen R, Gulliver M. Neonatal lupus erythematosus progressing into systemic lupus erythematosus. $\mathrm{Br} J$ Dermatol 1979;101:81-6.
}

Correspondence to Dr R N Srivastava, Department of Paediatrics, All India Institute of Medical Sciences, New Delhi 110 029, India.

Received 4 December 1986

\title{
Multiple thromboses in systemic lupus erythematosus
}

\author{
S APPAN, M L BOEY, AND K W LIM \\ Department of Paediatrics, Tan Tock Seng Hospital, Singapore
}

SUMMARY A 5 year old girl with systemic lupus erythematosus developed extensive thromboses at multiple sites. Coagulation screen confirmed presence of the lupus anticoagulant. She recovered satisfactorily on high dose steroids without the use of anticoagulant treatment.

Patients with the lupus anticoagulant are known to have an increased tendency to develop thromboembolic complications. This has been reported only rarely, however, in young children. We report a case of extensive thrombosis in a 5 year old girl with systemic lupus erythematosus and autoimmune haemolytic anaemia.

\section{Case report}

A 5 year old Chinese girl first presented with a history of fever and progressive pallor for one month. Clinical examination revealed striking pallor, bilateral cervical lymphadenopathy, and hepatosplenomegaly.

Laboratory investigations showed a haemoglobin concentration of $3.7 \mathrm{~g} / \mathrm{dl}$, white blood cell count $16.9 \times 10^{9} / 1$ (polymorphs $79 \%$, lymphocytes $16 \%$, monocytes $2 \%$, eosinophils $1 \%$, and myelocytes $2 \%$ ), platelets $105 \times 10^{9} / 1$, reticulocytes $40 \%$, and erythrocyte sedimentation rate $170 \mathrm{~mm}$ in the first hour. Direct Coombs test yielded strongly positive results. Rheumatoid factor and lupus erythematosus cells were negative. Serum complement (total complement CH50) was 13 units (normal 30-40 units). Antinuclear antibody and anti-deoxyribonucleic acid (anti-DNA) (double stranded) antibody (haemagglutination test 1/32) were both positive (significant at $1 / 4$ and above). Complement binding IgG autoantibody was detected in the serum. Blood urea, electrolyte, and creatinine concentrations were normal. Urine microscopy yielded normal results.

A diagnosis of systemic lupus erythematosus with autoimmune haemolytic anaemia was made. The patient was treated with hydrocortisone and transfused with washed packed cells. She was discharged well, on treatment with oral prednisolone. After one month on a dosage of $2 \mathrm{mg} / \mathrm{kg} /$ day her blood counts were haemoglobin $12 \mathrm{~g} / \mathrm{dl}$, erythrocyte sedimentation rate $10 \mathrm{~mm}$ in the first hour, serum complement 33 units, and antinuclear and anti-DNA antibodies negative. A direct Coombs test yielded negative results. The steroid dosage was gradually tailed down to $5 \mathrm{mg}$ every other day and she was maintained on this dose for one year. As she remained well both clinically and serologically and had no other organ involvement the steroid dosage was further tailed down and finally stopped. She was followed up closely and her blood counts and serological markers were within normal limits.

Three months after the treatment with steroids had been stopped, however, she relapsed. Her haemoglobin concentration was $8.8 \mathrm{~g} / \mathrm{dl}$, white blood cell count $8 \cdot 7 \times 10^{9} / 1$, platelets $15 \times 10^{9} / 1$, reticulocytes $3 \%$, and erythrocyte sedimentation rate $130 \mathrm{~mm}$ in the first hour. Direct Coombs test yielded positive results. Antinuclear and anti-DNA antibodies were both negative. There was no haematuria or proteinuria to suggest any renal involvement. She was restarted on prednisolone at $2 \mathrm{mg} / \mathrm{kg} /$ day.

A week later she developed focal convulsions of left upper and lower limbs. Blood pressure was systolic $100 \mathrm{~mm} \mathrm{Hg}$ and diastolic $60 \mathrm{~mm} \mathrm{Hg}$. A computed tomogram of the brain showed an infarct in the right frontoparietal region. Lumbar puncture showed normal cerebrospinal fluid. She was thus diagnosed as having cerebral lupus and was pulsed with methylprednisolone $200 \mathrm{mg}$ daily for three days 
and then continued with oral prednisolone and phenobarbitone. Three days later she developed peripheral venous thrombosis of both hands and feet. Fundoscopy of the left eye showed the retinal veins to be engorged and tortuous with multiple flame shaped retinal haemorrhages-a picture consistent with retinal vein thrombosis. Her right eye was normal. The lupus anticoagulant was detected by the method of Boey et al, ${ }^{1}$ which is a modified mixing partial thromboplastin time with kaolin. The anticoagulant is considered present when a prolonged kaolin partial thromboplastin time is not corrected by the addition of normal plasma. The upper limit of normal is five seconds more than the control, above which the test is considered to be positive. This test was positive in our patient as 50:50 mixture of normal and patient's plasma was $166 \cdot 2$ seconds (control 70.4 seconds). Anti-thrombin 3 was assayed by both functional and immunological methods and the results were normal. The parents refused anticoagulant treatment.

Therefore she was treated only with high dose steroids and antibiotics. Fasciotomy and decompression were performed for the affected limbs. Postoperatively, all the limbs except the right hand improved. The fingers of the right hand became gangrenous (Figure). The parents refused amputation. The gangrenous fingers gradually self amputated, leaving behind a stump, at the metacarpophalangeal joint. Haemoglobin concentration, platelet count, and erythrocyte sedimentation rate returned to normal and results of direct Coombs test became negative. She was discharged after four weeks of admission to hospital on phenobarbitone $30 \mathrm{mg}$ twice a day and prednisolone $15 \mathrm{mg}$ once a day.



Figure Progressive gangrene of the fingers and dorsum of the right hand despite fasciotomy and decompression.
Treatment was tailed down gradually and she is now on alternate day prednisolone $(5 \mathrm{mg}$ every other day). The lupus anticoagulant factor remained positive in her serum a month after discharge. Her condition has remained stable apart from blindness of the left eye and the self amputated stump of the right hand.

\section{Discussion}

Our patient was diagnosed as having systemic lupus erythematosus at a very young age of 4 years, with autoimmune haemolytic anaemia as the presenting manifestation. A year later she developed extensive thromboses, involving the retinal veins, superficial veins of hands and feet, and most probably the cerebral veins as well, manifesting as cerebral infarction. Although thrombosis is a well recognised complication of systemic lupus erythematosus and is known to occur in renal, retinal, cerebral, pulmonary, and leg veins, it is rare for it to affect multiple sites simultaneously as it did in our patient. The coagulation screen confirmed the presence of circulating lupus anticoagulant.

The lupus anticoagulant is an acquired inhibitor of the prothrombin complex (factor XA, factor $\mathrm{V}$, calcium, and phospholipid). ${ }^{2}$ It is an immunoglobulin of either the IgG or IgM or a mixed IgG and IgM group. Carreras et al have shown that the gammaglobulin fraction from patients with the lupus anticoagulant inhibits production of prostacyclin by vascular endothelial cells. ${ }^{3}$ Lower prostacyclin concentrations may result in increased platelet aggregation and thrombosis.

Patients with circulating lupus anticoagulant, paradoxically, have increased thrombotic activity, and bleeding tendency seems to be uncommon, except in cases with coexisting thrombocytopenia. ${ }^{4}$

Steroids, non-steroidal anti-inflammatory drugs, and cytotoxic agents have been used by others without any effect on the presence of the lupus anticoagulant. ${ }^{5}$ Other modalities of treatment are anticoagulant treatment and plasma exchange. There have been insufficient data to conclude about the efficacy of such forms of treatment. Use of prostacyclin rather than anticoagulants in acute management is worthy of consideration. Intravenous prostacyclin has been used in severe Raynaud's phenomenon. To date there has been no report of its use in systemic lupus erythematosus with the lupus anticoagulant. Our patient responded well to treatment with steroids and has remained in a satisfactory condition on low dose steroids. She has, however, a possible long term risk of having repeated spontaneous abortions and intrauterine deaths when she reaches reproductive age. ${ }^{6}$ 


\section{References}

1 Boey ML, Colaco CB, Gharavi AE, Elkon KB, Loizou S, Hughes GRV. Thrombosis in systemic lupus erythematosus: striking association with the presence of circulating lupus anticoagulant. Br Med J 1983;287:1021-3.

2 Exner T, Rickard KA, Kronenberg H. Studies on phospholipids in the actions of a lupus coagulation inhibitor. Pathology 1975;7:319-28.

${ }^{3}$ Carreras LO, Defreyn G, Machin SJ, et al. Arterial thrombosis, intrauterine death and the "lupus" anticoagulant detection of immunoglobin interfering with prostacyclin formation. Lancet $1981 ; \mathrm{i}: 244$.
4 Shapiro SS, Thiagarajan P. Lupus anticoagulants. Prog Hemost Thromb 1982;6:263-85.

${ }^{5}$ Mazen E, Amiram E. Thromboembolism in patients with the 'lupus'-type, circulating anticoagulant. Arch Intern Med 1984;144:510-15.

${ }^{6}$ Lubbe WF, Palmar SJ, Butler WS, Laggins GC. Fetal survival after prednisolone suppression of maternal lupus anticoagulant. Lancet 1983; i:1361-3.

Correspondence to Dr S Appan, Department of Paediatrics, Singapore General Hospital, Outram Road, Singapore 0316.

Received 5 January 1987

\title{
Swivel walkers in Duchenne muscular dystrophy
}

\author{
J R SIBERT, V WILLIAMS, R BURKINSHAW, AND S SIBERT
}

Llandough Hospital, Penarth, South Glamorgan, University Hospital of Wales, Cardiff, and Pentwyn School, Cardiff, Wales

SUMMARY Swivel walkers were used to provide low energy ambulation in 11 boys with Duchenne muscular dystrophy in schools for the physically handicapped in South Glamorgan. Our preliminary experience suggests that these walkers improve the quality of life and provide a useful part of the physical treatment of the condition.

There are clear advantages in prolonging the mobilisation of boys with Duchenne muscular dystrophy, most particuarly in improving morale. The use of long leg calipers was first advocated by Spencer and Vignos in the United States ${ }^{1}$ and has been used in the United Kingdom, particularly at the Hammersmith Hospital. ${ }^{2}$ Nevertheless, this approach has not been universally accepted. There have been difficulties with stability, with the boys falling over easily, fitting can be complicated, and the period that the walking is prolonged can be a short one.

Swivel walkers were first introduced in America for children with amelia. The principle of their use is a pylon arrangement that uses side to side oscillation together with a forward centre of gravity to provide ambulation. Their use for children with a paraplegia was pioneered in the United Kingdom by Rose. ${ }^{3}$ Their use for patients with muscular dystrophy was first suggested in the United Kingdom in 1981, providing a means of low energy ambulation for the boys, and has been developed by Bennett. ${ }^{4}$ We describe our preliminary experience with swivel walkers in boys with Duchenne muscular dystrophy in schools for the physically handicapped in South Glamorgan.

\section{Methods and results}

Most of the boys with Duchenne muscular dystrophy at the special schools for the physically handicapped in South Glamorgan (Pentwyn and Erw'r Delyn) have actively chosen to use swivel walkers as part of a programme of physical treatment for their condition. This programme has been part of a multidisciplinary, problem orientated approach to muscle disease centred at the muscle clinic and regional genetic centre at the University Hospital of Wales (Professor P Harper and Dr J R Sibert). As well as dealing with the physical problems we offer a comprehensive genetic service and active help in resolving any problems in the home situation.

In 1984 swivel walkers were introduced into the special schools and up to now have been used by 11 boys. Only one boy had declined to participate because he felt unwilling to have the extra physiotherapy involved. We have used two types of swivel walkers: initially, we used the Shrewsbury type, but more recently we have had good experience with the Salford swivel walker. We have reason to believe that the Orlav swivel walker would also be satisfactory. Elective operations on the Achilles tendons have been performed in most boys to help wheelchair position and comfort and to help with the use of the swivel walkers.

The boys are placed in their swivel walkers by physiotherapists and helpers and are encouraged to walk. It takes two people about three minutes to place a boy in a walker. The boys can use their swivel walkers to go between classes or can be taken 